\title{
Magnetic Centres in Functionalized Graphene
}

\author{
Ł. Majchrzycki ${ }^{a, b, *}$, M.A. Augustyniak-JAblokOW ${ }^{c}$, R. StrzelczYK ${ }^{c}$, \\ AND M. MAĆKOWIAK ${ }^{c}$ \\ ${ }^{a}$ Institute of Physics, Poznań University of Technology, Nieszawska 13a, 60-965 Poznań, Poland \\ ${ }^{b}$ Wielkopolska Centre of Advanced Technology, Adam Mickiewicz University, Grunwaldzka 6, 60-780 Poznań, Poland \\ ${ }^{c}$ Institute of Molecular Physics, Polish Academy of Sciences, M. Smoluchowskiego 17, 60-179 Poznań, Poland
}

Discussion of the origin of paramagnetic centres observed by electron paramagnetic resonance in graphene oxide (GO) and reduced graphene oxide (rGO) is done on the assumption that GO can be considered as a functionalized graphene. This leads to the conclusion that the narrow signal with $g$ close to 2, observed for GO and thermally reduced GO, is due to paramagnetic centres localized on defects and exchange coupled to conduction electrons. Randomness of graphene modification results in variety of parameters of EPR signals. The broad signals observed in GO and rGO and ascribed to magnetic clusters on the zig-zag edge states indicate that the edge magnetism can be preserved by functionalization.

DOI: 10.12693 /APhysPolA.127.540

PACS: 75.70.Ak, 81.05.ue, 76.50.+g

\section{Introduction}

The aim of graphene functionalization is to change its properties in order to stabilize graphene sheets in various media [1], to produce a semiconducting material by opening the energy gap, as well as to tailor graphene for biomedical applications [2]. Functionalization can be intentionally introduced, but it is also the inevitable effect of production processes. As a result, paramagnetic centres appear in such materials due to presence of functional groups or local instabilities of the electronic structure of graphene.

The chemical route to graphene $[3,4]$ starts from oxidation of graphite in strong acids resulting in graphite intercalation by the epoxy and hydroxyl $(\mathrm{OH})$ groups attached to the graphene surface as well as the carboxyl groups bounded to the edges. This increases the distance between graphene layers and makes graphene layers hydrophilic and able to exfoliate in water. Study of graphene oxide (GO) by scanning transmission electron microscopy combined with electron energy loss spectroscopy [5] and other experimental techniques supported by density functional theory calculation [6], show that graphene oxide can be considered as graphene functionalized by oxygen containing groups. Although the functional groups are not paramagnetic, electron paramagnetic resonance (EPR) study of GO reveals presence of a narrow signal, whose shape and temperature dependence varies on the production method, as well as on the graphene flake diameter [7-9].

Various methods of GO reduction, i.e. of functional groups removal are used $[10,11]$. Chemical methods are supposed to smoothly remove the functional groups, leaving undamaged graphene surface. Chemical analysis of

*corresponding author; e-mail: lukasz.majchrzycki@gmail.com the material reduced by hydrazine confirms removal of all oxygen but presence of a small admixture of nitrogen indicates that some hydrazine or products of hydrazine decomposition remains attached to the graphene. Theoretical considerations indicate some functionalization of the graphene edges. Such material is not expected to possess any magnetic moments, as zig-zag edges become passivated in the contact with atmospheric oxygen. The disadvantage of this method is strong agglomeration of the hydrophobic graphene flakes.

Thermal reduction of graphite oxide [6] amalgamates two processes. Removal of the functional groups attached to the graphene sheets increases the gas pressure inside the material, and results in a rapid exfoliation. However, removal of epoxy groups produces carbon vacancies and as a result graphene flakes are small, strongly defected and bent. These damages can be minimized if graphite oxide is first exfoliated in liquid. Thermal reduction of graphene oxide, which is produced in this way, requires much higher energy output. Obtaining high $\mathrm{C} / \mathrm{O}$ ratio is possible only in high temperatures using vacuum or reducing atmosphere. Such material is, usually, rich in attached atoms and defects, which introduces electron instabilities and results in the appearance of the paramagnetic centres.

The present study investigates the electronic structure of functionalized graphene. In this short report we restrain ourselves to results obtained for GO, hydrazine reduced GO (rGOH), GO reduced thermally in air (rGOT) and in paraffin (rGOp).

\section{Material and methods}

GO was obtained from graphite flakes by a modified Hummers method [4]. Sodium nitrite was dissolved into $95 \%$ sulphuric acid, then graphite flakes (Lonza KS 5$75 \mu \mathrm{m})$ were gradually added to the solution under a vigorous stirring. The mixture was cooled to $0^{\circ} \mathrm{C}$ in an 
ice bath, and left for $1.5 \mathrm{~h}$ under magnetic stirring. Then, under stirring, $\mathrm{KMnO}_{4}$ was gradually added, to avoid sudden temperature increase above $10^{\circ} \mathrm{C}$. Subsequently, the suspension was left for one week at the room temperature to allow acid intercalation into graphite. Then, the temperature of the mixture was increased to $35^{\circ} \mathrm{C}$ and stirred for 30 min before the distilled water was added to the paste. Subsequently temperature was increased to $98^{\circ} \mathrm{C}$ and maintained for $15 \mathrm{~min}$. Next, the suspension was diluted with distilled water and the same amount of $3 \%$ hydrogen peroxide. The residual manganese ions were removed by mixing the suspension into large amount of $1.3 \% \mathrm{HCl}$. The presence of sulphate ions was checked with barium chloride and the purifying process was conducted until the test result was negative. Next, the purification process was conducted by using distilled water until $\mathrm{pH}$ became neutral. Finally, the product was vacuum filtrated and dried in desiccator.

Another portion of product was centrifuged to obtained thick suspension, and after subsequent dilution was exfoliated by ultrasounds to a single-layer graphene oxide. Chemically processed $\mathrm{rGOH}$ was obtained by the hydrazine reduction of GO water solution. rGOT was produced by heating the dried GO in a furnace in air atmosphere up to $220^{\circ} \mathrm{C}$ for $1 \mathrm{~h}$.

Samples for EPR study were prepared by gradual deposition of aqueous (GO) or alcohol (rGO) suspensions on $\mathrm{MgO}$ powder. $\mathrm{GO}$ samples were also studied in the form of a film and of a graphene suspension obtained by thermal reduction of GO in a paraffin matrix ( $\mathrm{rGOp}$ ).

EPR measurements were performed on the X-band RADIOPAN SX spectrometer with Oxford CF935 cryostat allowing the measurements in the temperature range of $4.2-300 \mathrm{~K}$.

\section{Results and discussion}

Graphite and graphene oxide are precursors of all other samples studied by us. EPR spectra of these materials are gathered in Fig. 1. The spectrum of graphite oxide is represented by weak and narrow $(0.195 \mathrm{mT})$ signal of Lorentzian shape (Fig. 1a). But spectrum of a single layer graphene oxide deposited on $\mathrm{MgO}$ is not only weak, but also much broader $(0.47 \mathrm{mT})$ and slightly asymmetric (Fig. 1b). For the same material, in the form of GO film, the EPR signal is narrow again, but shows not only asymmetry, but is accompanied by satellite lines (Fig. 1c). Their separation, of about of $0.9 \mathrm{mT}$, indicates that these are the flip-flop signals due to nearby protons. Signal asymmetry increases at low temperatures (Fig. 1d), but its intensity very weakly depends on temperature. Signal amplification (20 times) and the extension of the magnetic field range revealed a broad signal with $g \approx 2.18$ shown in Fig. 2a and b. Materials prepared and studied by us are free from $\mathrm{Mn}^{2+}$ ions, which were suggested as a possible source of this signal [12].

In our approach we treat graphene oxide as a strongly defected graphene, and not as a new chemical compound.

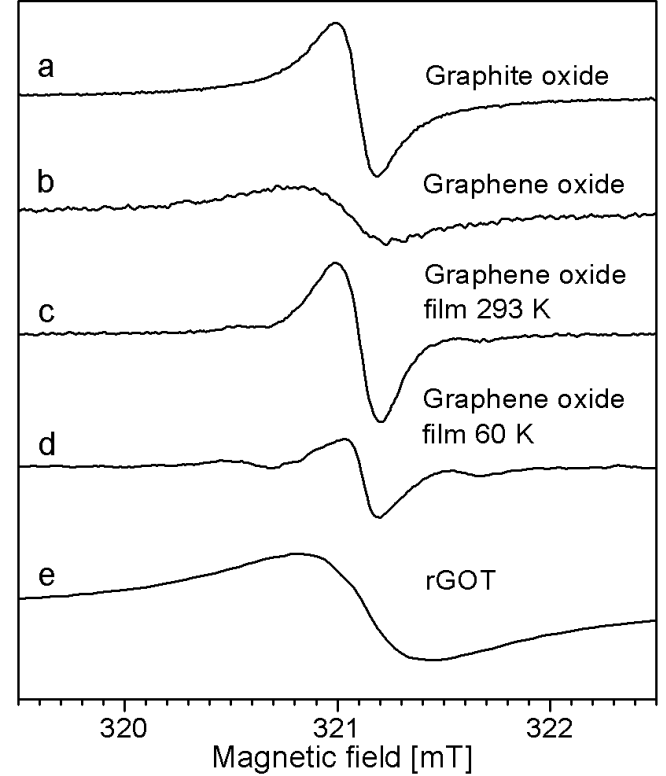

Fig. 1. EPR spectra of: (a) graphite oxide, (b) graphene oxide deposited on $\mathrm{MgO}$, (c) and (d) graphene oxide film at $293 \mathrm{~K}$ and $60 \mathrm{~K}$ respectively, (e) thermally reduced graphite oxide. Modulation amplitude $0.05 \mathrm{mT}$.

Under conditions of EPR experiment (low magnetic field, absence of the electric field) the signal of the ideal graphene can originate from the zig-zag edge states only. The real graphene is more or less disordered, part of defects is paramagnetic, and those, with energy close to the Fermi level can dope electrons to the conduction band $[13,14]$.

As graphite/graphene oxide, or more precisely, their films, show very high DC resistivity, one can expect very broad gap between valence and conduction bands. But our results as well as earlier finding of Ćirić et al. [7] indicate presence of centres, which due to Pauli-like temperature dependence can be identified with conduction electrons. Therefore, the insulating properties of GO can be due to disorder and the ordered, graphene-like zones, separated by highly disordered areas are supposed to exist in GO structure [5]. Existence of conducting electrons in these zones indicate changes in the structure of graphene energy levels.

When our results are consistent with those published by Ćirić et al. $[7,8]$ the $K$-band spectra, published by Rao et al. [9] indicate presence of two signals with $g \approx 2$. Differences in so far reported EPR spectra [7-9] can be explained by the fact that GO is randomly functionalized. Therefore, density of the localized centres as well as dimensions, and properties of graphene-like areas can vary from sample to sample, depending on graphite properties and oxidation process. Exchange interactions between localized and delocalized electrons, which strongly depend on the density of both types of paramagnetic centres, can additionally modify EPR spectra. 
The EPR signal of the thermally reduced graphite, (rGOT) shown in Fig. 1e, is broader than that of GO. Presence of this signal is not a surprise as the rGOT is only partly reduced and removed functional groups are substituted by carbon vacancies. As the energy of the unpaired electrons trapped on these vacancies is close to the Fermi level, a doping of electrons to the conduction band in the graphene-like areas is possible. Interpretation of this spectrum is, therefore, the same as that of GO.

Thermal reduction in paraffin results in much weaker signal at $g \approx 2$ (Fig. 2e), suggesting that the process of reduction is more subtle than in air.

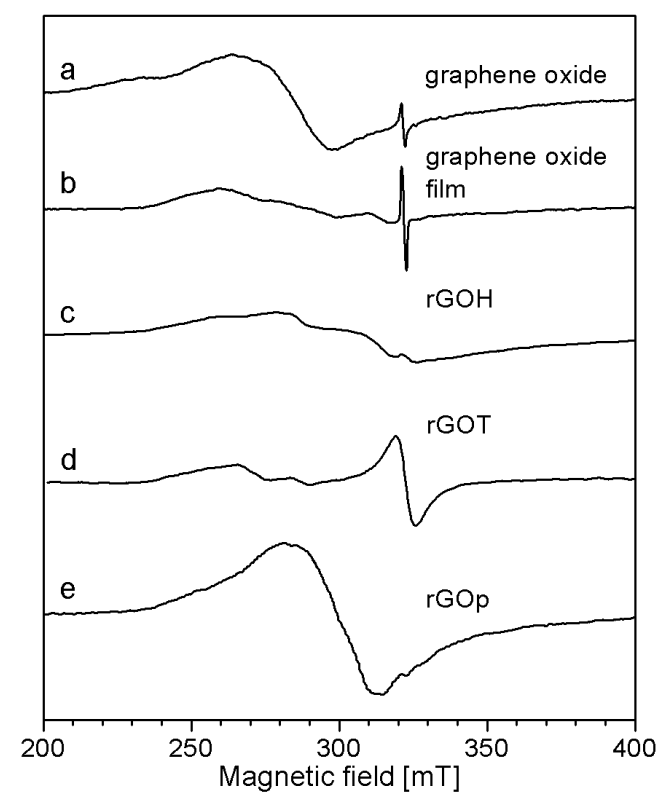

Fig. 2. EPR spectra of (a) graphene oxide deposited on $\mathrm{MgO}$, (b) graphene oxide film, (c) hydrazine reduced graphene oxide, (d) thermally reduced graphite oxide, (e) graphene oxide thermally reduced in paraffin. Modulation amplitude $1 \mathrm{mT}$.

The weakest signal at $g \approx 2$ is observed in the hydrazine reduced $\mathrm{rGOH}$. Here, only weak kink is visible in the overmodulated spectra shown in Fig. 2c. Instead a broad signal is revealed. Such broad signals are observed for all samples, and are especially intensive for $\mathrm{rGOp}-$ the sample reduced in paraffin (Fig. 2e). That signal can be ascribed to the ferromagnetically coupled spins on zig-zag edges [15], which at room temperature forms small magnetic clusters. Presence of this signal is understandable, as the paraffin matrix protects edges from contact with air. However, presence of similar signals in samples stored in air proves that some functionalization can preserve edge magnetism. In case of $\mathrm{rGOH}$ it can be hydrazine groups left attached to edges after reduction. In GO and rGOT such role can only be played by carboxyl groups. Although efficiency of such functionalization is weak, it indicates new methods for preserving edge magnetism of graphene.

\section{Conclusions}

Analysis of the EPR spectra of GO and rGO, made on the assumption that GO is a functionalized graphene, leads to conclusions that: (i) functionalization induces both localized paramagnetic centres and conduction electrons; (ii) these two types of paramagnetic centers are exchange coupled; (iii) weak, broad signals observed for $\mathrm{GO}$ and rGO indicate that functionalization can preserve edge magnetism.

\section{References}

[1] M. Song, D. Cai, in: Polymer-Graphene Nanocomposites, Ed. V. Mittal, RSC Publ., Dorchester 2012, p. 1.

[2] V. Georgakilas, M. Otyepka, A.B. Bourlinos, V. Chandra, N. Ki, K.C. Kemp, P. Hobza, R. Zboril, K.S. Kim, Chem. Rev. 112, 6156 (2012).

[3] Y. Zhu, S. Murali, W. Cai, X. Li, J.W. Suk, J.R. Potts, R.S. Ruoff, Adv. Mater. 22, 3906 (2010).

[4] W.S. Hummers Jr, R.E. Offeman, J. Am. Chem. Soc. 80, 1339 (1958).

[5] K.A. Mkhoyan, A.W. Contryman, J. Silcox, D.A. Stewart, G. Eda, C. Mattevi, S. Miller, M. Chhowalla, Nano Lett. 9, 1058 (2009).

[6] S. Pei, H.M. Cheng, Carbon 50, 3210 (2012).

[7] L. Ćirić, A. Sienkiewicz, D.M. Djokić, R. Smajda, A. Magrez, T. Kaspar, R. Nesper, L. Forró, Phys. Status Solidi B 247, 2958 (2010).

[8] L. Ćirić, A. Sienkiewicz, R. Gaál, J. Jaćimović, C. Vâju, A. Magrez, L. Forró, Phys. Rev. B 86, 195139 (2012).

[9] S.S. Rao, A. Stesmans, Y. Wang, Y. Chen, Physica E Low Dimens. Syst. Nanostruct. 44, 1036 (2012).

[10] X. Gao, J. Jang, S. Nagase, J. Phys. Chem. C 114, 832 (2009).

[11] C.K. Chua, M. Pumera, Chem. Soc. Rev. 43, 291 (2014).

[12] A.M. Panich, A.I. Shames, N.A. Sergeev, Appl. Magn. Reson. 44, 107 (2013).

[13] M.A. Augustyniak-Jabłokow, K. Tadyszak, M. Maćkowiak, Y.V. Yablokov, Phys. Status Solidi (RRL) - Rapid Res. Lett. 5, 271 (2011).

[14] M.A. Augustyniak-Jabłokow, K. Tadyszak, M. Maćkowiak, S. Lijewski, Chem. Phys. Lett. 557, 118 (2013).

[15] M.A. Augustyniak-Jabłokow, M. Maćkowiak, K. Tadyszak, R. Strzelczyk, Acta Phys. Pol. A 127, 537 (2015). 